\title{
Article
}

\section{Steroidal Carboxylic Acids from Soft Coral Paraminabea acronocephala}

\section{Chih-Hua Chao ${ }^{1,2,3}$, Yang-Chang Wu ${ }^{4}$, Zhi-Hong Wen ${ }^{1,5}$ and Jyh-Horng Sheu ${ }^{1,5}, *$}

1 Department of Marine Biotechnology and Resources, National Sun Yat-sen University, Kaohsiung 80424, Taiwan; E-Mails: chaochihhua@hotmail.com (C.-H.C.); wzh@mail.nsysu.edu.tw (Z.-H.W.)

2 Chinese Medicine Research and Development Center, China Medical University Hospital, Taichung 40402, Taiwan

3 China Medical University, Taichung 40402, Taiwan

4 Graduate Institute of Integrated Medicine, College of Chinese Medicine, China Medical University, Taichung 40402, Taiwan; E-Mail: yachwu@mail.cmu.edu.tw

5 Asia-Pacific Ocean Research Center, National Sun Yat-sen University, Kaohsiung 80424, Taiwan

* Author to whom correspondence should be addressed; E-Mail: sheu@mail.nsysu.edu.tw; Tel.: +886-7-5252000 (ext. 5030); Fax: +886-7-5255020.

Received: 28 November 2012; in revised form: 28 December 2012 / Accepted: 31 December 2012 / Published: 11 January 2013

Abstract: Three new steroidal carboxylic acids, paraminabic acids A-C (1-3) were isolated from a Formosan soft coral Paraminabea acronocephala. The structures of these compounds were established by extensive spectroscopic analysis and chemical methods. Application of the PGME method allowed the establishment of the absolute configurations at C-25 and C-24 for $\mathbf{1}$ and $\mathbf{2}$, respectively. Compound $\mathbf{3}$ showed potent cytotoxicity toward Hep3B, MDA-MB-231, MCF-7, and A-549 cancer cell lines, with $\mathrm{IC}_{50}$ values ranging from 2.05 to $2.83 \mu \mathrm{g} / \mathrm{mL}$. Compounds 2 and 3 were found to inhibit the accumulation of the pro-inflammatory iNOS protein.

Keywords: Paraminabea acronocephala; paraminabic acid; soft coral; cytotoxicity; anti-inflammatory activity 


\section{Introduction}

Marine withanolides, with potent pro-inflammatory inducible nitric oxide synthase (iNOS) inhibitory activity, have previously been reported from two species of soft corals, Paraminabea acronocephala [1] and Minabea sp. [2]. These compounds possess a different A-ring structure (1,4-dien-3-one or 4-en-3-one) from those of plant origin [1-3]. Our previous chemical investigation of the soft coral $P$. acronocephala led to the isolation of novel withanolides with a $24 \beta, 25 \beta$-dimethyl- $\gamma$-lactone or a $24 \beta, 25 \alpha$-dimethyl- $\gamma$-lactone in the steroidal side chain moiety [1]. As part of our continuing search for bioactive, structurally interesting metabolites from this coral, three steroidal carboxylic acids (1-3) were isolated and their structures were elucidated (Figure 1). The cytotoxicity of compounds $\mathbf{1}-\mathbf{3}$ against human liver carcinoma (HepG2 and HepG3), human breast carcinoma (MCF-7 and MDA-MB-231), and human lung carcinoma (A-549) cell lines and the ability of 1-3 to inhibit up-regulation of the pro-inflammatory iNOS and COX-2 (cyclooxygenase-2) proteins in LPS (lipopolysaccharide)-stimulated RAW264.7 macrophage cells were also evaluated.

Figure 1. The structures of paraminabic acids $\mathrm{A}-\mathrm{C}(\mathbf{1}-\mathbf{3})$.

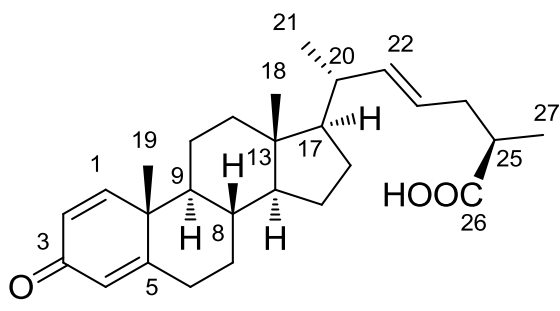

paraminabic acid $A(1)$

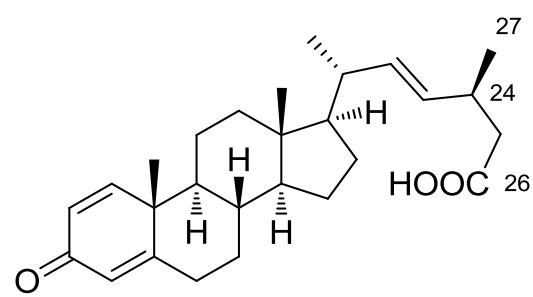

paraminabic acid $B(2)$

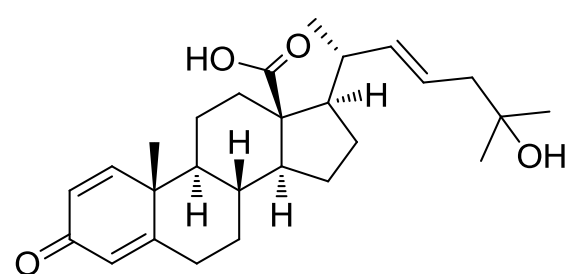

paraminabic acid C (3)

\section{Results and Discussion}

The ethanolic extract of the soft coral $P$. acronocephala was partitioned between EtOAc and $\mathrm{H}_{2} \mathrm{O}$ to afford the EtOAc-soluble fraction. It was then subjected to silica gel column chromatography. The fractions containing steroids were selected for further study, based on characteristic methyl signals in the ${ }^{1} \mathrm{H}$ NMR spectrum. These fractions were subsequently subjected to a series of chromatographic separations to afford three new steroidal carboxylic acids, paraminabic acids A-C (1-3).

The HRESIMS and ${ }^{13} \mathrm{C}$ NMR spectroscopic data of paraminabic acid A (1) suggested a molecular formula of $\mathrm{C}_{27} \mathrm{H}_{38} \mathrm{O}_{3}$, appropriate for nine degrees of unsaturation. The ${ }^{13} \mathrm{C} \mathrm{NMR}$ and DEPT spectroscopic data (Table 1) displayed 27 carbon signals, including 4 methyls, 7 methylenes, 11 methines, and 5 quaternary carbons. A broad $\mathrm{O}-\mathrm{H}$ stretching absorption in the region of $3400-2600 \mathrm{~cm}^{-1}$ is ascribable to a carboxylic acid, which was evidenced by the carbon resonance at $\delta_{\mathrm{C}} 180.6(\mathrm{C})$. The same steroidal nucleus as that of paraminabeolides $\mathrm{D}$ and $\mathrm{E}$ was deduced for $\mathbf{1}$ by detailed comparison of their NMR spectroscopic data [1]. The side chain moiety of 1 resembles that of a known steroidal carboxylic acid, (25S)-3-oxocholesta-1,4-dien-26-oic acid [4], isolated from the Indonesian soft coral Minabea sp. However, 1 varied from (25S)-3-oxocholesta-1,4-dien-26-oic acid in the respective side chain. The proton resonances at $\delta_{\mathrm{H}} 5.50(1 \mathrm{H}, \mathrm{dt}, J=15.6,6.4 \mathrm{~Hz}, \mathrm{H}-23)$ and $5.44(1 \mathrm{H}, \mathrm{dd}, J=15.6,8.8 \mathrm{~Hz}, \mathrm{H}-22)$, measured in $\mathrm{C}_{5} \mathrm{D}_{5} \mathrm{~N}$, were due to the presence of a trans C-22/C-23 double bond, which was confirmed by the HMBC correlations from $\mathrm{H}_{3}-21$ to $\mathrm{C}-17$, C-20, 
and $\mathrm{C}-22$. The absolute configuration at C-25 was determined by the application of Kusumi's method (PGME method) [5-7]. The chemical shift differences of ( $S$ )-PGME amide (1a) and $(R)$-PGME amide (1b) $\left(\Delta \delta=\delta_{(S)}-\delta_{(R)}\right)$ were summarized in Figure 2 and established the $R$ configuration at C-25.

Table $1 .{ }^{13} \mathrm{C}$ NMR spectroscopic data of compounds $\mathbf{1}-\mathbf{3}$.

\begin{tabular}{|c|c|c|c|}
\hline Position & $1^{\mathrm{a}}, \delta_{\mathrm{C}}$, mult. & $2^{a}, \delta_{C}$, mult. & $3^{\mathrm{a}}, \delta_{\mathrm{C}}$, mult. \\
\hline 1 & 156.1, CH & 156.1, CH & $155.9, \mathrm{CH}$ \\
\hline 2 & $127.4, \mathrm{CH}$ & 127.4, CH & $127.5, \mathrm{CH}$ \\
\hline 3 & $186.5, \mathrm{C}$ & $186.5, \mathrm{C}$ & $186.5, \mathrm{C}$ \\
\hline 4 & $123.8, \mathrm{CH}$ & 123.7, $\mathrm{CH}$ & $123.8, \mathrm{CH}$ \\
\hline 5 & 169.5, C & 169.6, C & 169.3, C \\
\hline 6 & $32.9, \mathrm{CH}_{2}$ & $32.9, \mathrm{CH}_{2}$ & $32.7, \mathrm{CH}_{2}$ \\
\hline 7 & $33.7, \mathrm{CH}_{2}$ & $33.7, \mathrm{CH}_{2}$ & $33.4, \mathrm{CH}_{2}$ \\
\hline 8 & $35.5, \mathrm{CH}$ & $35.5, \mathrm{CH}$ & $37.1, \mathrm{CH}$ \\
\hline 9 & $52.4, \mathrm{CH}$ & $52.4, \mathrm{CH}$ & $52.4, \mathrm{CH}$ \\
\hline 10 & 43.6, C & 43.6, C & 43.6, C \\
\hline 11 & $22.8, \mathrm{CH}_{2}$ & $22.8, \mathrm{CH}_{2}$ & 24.6, $\mathrm{CH}_{2}$ \\
\hline 12 & $39.3, \mathrm{CH}_{2}$ & $39.3, \mathrm{CH}_{2}$ & $35.1, \mathrm{CH}_{2}$ \\
\hline 13 & $42.6, \mathrm{C}$ & $42.5, \mathrm{C}$ & $55.8, \mathrm{C}$ \\
\hline 14 & $55.6, \mathrm{CH}_{2}$ & $55.5, \mathrm{CH}_{2}$ & $55.7, \mathrm{CH}_{2}$ \\
\hline 15 & 24.4, $\mathrm{CH}_{2}$ & 24.3, $\mathrm{CH}_{2}$ & 25.0, $\mathrm{CH}_{2}$ \\
\hline 16 & $28.4, \mathrm{CH}_{2}$ & 28.3, $\mathrm{CH}_{2}$ & 25.3, $\mathrm{CH}_{2}$ \\
\hline 17 & $55.5, \mathrm{CH}$ & $55.5, \mathrm{CH}$ & $55.3, \mathrm{CH}$ \\
\hline 18 & $12.2, \mathrm{CH}_{3}$ & $12.2, \mathrm{CH}_{3}$ & $176.8, \mathrm{C}^{\mathrm{b}}$ \\
\hline 19 & $18.7, \mathrm{CH}_{3}$ & $18.7, \mathrm{CH}_{3}$ & $18.7, \mathrm{CH}_{3}$ \\
\hline 20 & $40.0, \mathrm{CH}$ & $39.9, \mathrm{CH}$ & $38.4, \mathrm{CH}$ \\
\hline 21 & $20.6, \mathrm{CH}_{3}$ & $20.6, \mathrm{CH}_{3}$ & $22.0, \mathrm{CH}_{3}$ \\
\hline 22 & 139.6, $\mathrm{CH}$ & 136.1, CH & $136.7, \mathrm{CH}$ \\
\hline 23 & $123.8, \mathrm{CH}$ & 131.3, CH & $124.8, \mathrm{CH}$ \\
\hline 24 & $36.4, \mathrm{CH}_{2}$ & $33.7, \mathrm{CH}$ & $46.8, \mathrm{CH}_{2}$ \\
\hline 25 & $39.3, \mathrm{CH}$ & $41.6, \mathrm{CH}_{2}{ }^{\mathrm{b}}$ & 71.7, C \\
\hline 26 & $180.6, \mathrm{C}^{\mathrm{b}}$ & $176.9, \mathrm{C}^{\mathrm{b}}$ & 27.6, $\mathrm{CH}_{3}$ \\
\hline 27 & $16.3, \mathrm{CH}_{3}{ }^{\mathrm{b}}$ & 20.6, $\mathrm{CH}_{3}$ & $30.5, \mathrm{CH}_{3}$ \\
\hline
\end{tabular}

${ }^{\mathrm{a}}$ Spectra were measured in $\mathrm{CDCl}_{3}(100 \mathrm{MHz}) ;{ }^{\mathrm{b}}$ values obtained from the relevant $\mathrm{HMBC}$ or HSQC correlation peaks.

Paraminabic acid B (2) gave the same molecular formula, $\mathrm{C}_{27} \mathrm{H}_{38} \mathrm{O}_{3}$, as that of $\mathbf{1}$, based on the analysis of the HRESIMS and ${ }^{13} \mathrm{C}$ NMR spectroscopic data (Table 1). The NMR spectroscopic data of $\mathbf{2}$ are similar to those of $\mathbf{1}$, but differences were observed in their side chains. The HMBC correlations from $\mathrm{H}_{3}-21$ to $\mathrm{C}-17, \mathrm{C}-20$, and $\mathrm{C}-22$ allowed the assignment of a C-22/C-23 double bond. A large coupling constant $\left(15.2 \mathrm{~Hz}, \mathrm{C}_{5} \mathrm{D}_{5} \mathrm{~N}\right)$ between $\mathrm{H}-22$ and $\mathrm{H}-23$ suggested the $E$ geometry of this double bond. The H-23 signal appeared as a doublet of doublets, revealing that the adjacent carbon (C-24) should be a methine. This might be due to the attachment of a methyl group $\left(\delta_{\mathrm{H}} 1.03,3 \mathrm{H}, \mathrm{d}, J=6.4 \mathrm{~Hz}\right.$, $\mathrm{H}_{3}$-27) at C-24 (Table 2). This was confirmed by the HMBC correlations from $\mathrm{H}_{3}-27$ to C-23, C-24, and $\mathrm{C}-25$ as well as from $\mathrm{H}_{2}-25$ to the carboxyl carbon (C-26). The absolute configuration at C-24 of 2 
was determined by the application of Kusumi's method developed for chiral $\beta, \beta$-disubstituted propionic acid derivatives [6,7]. The ${ }^{1} \mathrm{H}$ NMR shift differences $\left(\Delta \delta=\delta_{(R)}-\delta_{(S)}\right)$ between the diastereomeric $(R)$ - and $(S)$-PGME amides, $\mathbf{2} \mathbf{a}$ and $\mathbf{2 b}$, respectively, are summarized in Figure 2 and establish the $24 S$ configuration for 2.

Table 2. ${ }^{1} \mathrm{H}$ NMR spectroscopic data of compounds 1-3.

\begin{tabular}{|c|c|c|c|}
\hline$\#$ & $1, \delta_{\mathrm{H}}(J \text { in } \mathrm{Hz})^{\mathrm{a}}$ & $2, \delta_{\mathrm{H}}(J \text { in } \mathrm{Hz})^{\mathrm{a}}$ & $3, \delta_{\mathrm{H}}(J \text { in Hz})^{\mathrm{a}}$ \\
\hline 1 & 7.06, d (10.0) & $7.06, \mathrm{~d}(10.0)$ & $7.03, \mathrm{~d}(10.0)$ \\
\hline 2 & $6.23, \mathrm{dd}(10.0,1.6)$ & $6.24, \mathrm{~d}(10.0)$ & $6.23, \mathrm{~d}(10.0)$ \\
\hline 4 & $6.07, \mathrm{~s}$ & $6.07, \mathrm{~s}$ & $6.07, \mathrm{~s}$ \\
\hline \multirow[t]{2}{*}{6} & $\mathrm{a}: 2.45, \mathrm{~m}$ & a: $2.46, \operatorname{td}(13.6,4.4)$ & a: $2.46, \operatorname{td}(13.4,4.4)$ \\
\hline & $\mathrm{b}: 2.35, \mathrm{~m}$ & $\mathrm{~b}: 2.35, \mathrm{~m}$ & $\mathrm{~b}: 2.35, \mathrm{~m}$ \\
\hline \multirow[t]{2}{*}{7} & $\mathrm{a}: 1.93, \mathrm{~m}$ & $\mathrm{a}: 1.93, \mathrm{~m}$ & $\mathrm{a}: 2.04, \mathrm{~m}$ \\
\hline & $\mathrm{b}: 1.02, \mathrm{~m}$ & $\mathrm{~b}: 1.02, \mathrm{~m}$ & $\mathrm{~b}: 1.07, \mathrm{~m}$ \\
\hline 8 & $1.60, \mathrm{~m}$ & $1.59, \mathrm{~m}$ & $1.69, \mathrm{~m}$ \\
\hline 9 & $1.04, \mathrm{~m}$ & $1.03, \mathrm{~m}$ & $1.10, \mathrm{~m}$ \\
\hline \multirow[t]{2}{*}{11} & $1.67, \mathrm{~m}$ & $1.67, \mathrm{~m}$ & $1.85, \mathrm{~m}$ \\
\hline & & & $1.69, \mathrm{~m}$ \\
\hline \multirow[t]{2}{*}{12} & $\mathrm{a}: 2.00, \mathrm{~m}$ & $\mathrm{a}: 1.99, \mathrm{~m}$ & a: 2.66, br d (14.0) \\
\hline & $\mathrm{b}: 1.18, \mathrm{~m}$ & $\mathrm{~b}: 1.17, \mathrm{~m}$ & $\mathrm{~b}: 1.03, \mathrm{~m}$ \\
\hline 14 & $1.12, \mathrm{~m}$ & $1.11, \mathrm{~m}$ & $1.30, \mathrm{~m}$ \\
\hline \multirow[t]{2}{*}{15} & $\mathrm{a}: 1.55, \mathrm{~m}$ & $\mathrm{a}: 1.52, \mathrm{~m}$ & $\mathrm{a}: 1.91, \mathrm{~m}$ \\
\hline & $\mathrm{b}: 1.08, \mathrm{~m}$ & $\mathrm{~b}: 1.02, \mathrm{~m}$ & $\mathrm{~b}: 1.66, \mathrm{~m}$ \\
\hline \multirow[t]{2}{*}{16} & $\mathrm{a}: 1.65, \mathrm{~m}$ & $\mathrm{a}: 1.62, \mathrm{~m}$ & $\mathrm{a}: 1.78, \mathrm{~m}$ \\
\hline & $\mathrm{b}: 1.22, \mathrm{~m}$ & $\mathrm{~b}: 1.20, \mathrm{~m}$ & b: $1.70, \mathrm{~m}$ \\
\hline 17 & $0.99, \mathrm{~m}$ & $0.99, \mathrm{~m}$ & $1.62, \mathrm{~m}$ \\
\hline 18 & $0.74, \mathrm{~s}$ & $0.74, \mathrm{~s}$ & \\
\hline 19 & $1.23, \mathrm{~s}$ & $1.23, \mathrm{~s}$ & $1.15, \mathrm{~s}$ \\
\hline 20 & $2.03, \mathrm{~m}$ & $2.00, \mathrm{~m}$ & $2.36, \mathrm{~m}$ \\
\hline 21 & $0.99, \mathrm{~d}(6.8)$ & $0.98, \mathrm{~d}(6.4)$ & $1.05, \mathrm{~d}(6.4)$ \\
\hline 22 & $5.27-5.30^{b}$ & $5.23-5.26^{b}$ & $5.39 \mathrm{dd}(15.2,8.8)$ \\
\hline 23 & $5.27-5.30^{b}$ & $5.23-5.26^{b}$ & 5.48, ddd $(15.2,8.8,5.2)$ \\
\hline \multirow[t]{2}{*}{24} & $\mathrm{a}: 2.33, \mathrm{~m}$ & $2.59, \mathrm{~m}$ & $2.18, \mathrm{dd}(14.0,5.2)$ \\
\hline & $\mathrm{b}: 2.10, \mathrm{~m}$ & & $2.11, \mathrm{dd}(14.0,8.8)$ \\
\hline 25 & $2.49, \mathrm{~m}$ & $2.30, \mathrm{~d}(7.2)$ & \\
\hline 26 & & & $1.25, \mathrm{~s}$ \\
\hline 27 & $1.15, \mathrm{~d}(7.2)$ & $1.03, \mathrm{~d}(6.4)$ & $1.25, \mathrm{~s}$ \\
\hline
\end{tabular}

The HRESIMS and ${ }^{13} \mathrm{C}$ NMR spectroscopic data of paraminabic acid C (3) established a molecular formula of $\mathrm{C}_{27} \mathrm{H}_{38} \mathrm{O}_{4}$ and nine degrees of unsaturation. The IR absorptions at 3419 and $1714 \mathrm{~cm}^{-1}$ suggested the presence of hydroxy and carbonyl groups, respectively. Both ${ }^{1} \mathrm{H}$ and ${ }^{13} \mathrm{C} \mathrm{NMR}$ spectra of 3 lacked signals of the angular methyl group, which might be replaced by a carboxylic acid according to the carbon signal at $\delta_{\mathrm{C}} 176.8$ (C) (Table 1). The carboxylic acid attached at $\mathrm{C}-13$ was further confirmed by the HMBC correlations from both $\mathrm{H}_{2}-12$ and $\mathrm{H}-17$ to $\mathrm{C}-18$. The trans $\mathrm{C}-22 / \mathrm{C}-23$ double 
bond was deduced by the HMBC correlations from $\mathrm{H}_{3}-21$ to $\mathrm{C}-17, \mathrm{C}-20$, and $\mathrm{C}-22$ as well as $J$ value $(15.2 \mathrm{~Hz})$ (Table 2) between $\mathrm{H}-22$ and H-23. In addition, the downfield-shifted quaternary carbon at $\delta_{\mathrm{C}} 69.9$ was ascribable to a hydroxy group attached at C-25, which was correlated by $\mathrm{H}_{2}-24, \mathrm{H}_{3}-26$, and $\mathrm{H}_{3}-27$ in the $\mathrm{HMBC}$ spectrum.

Figure 2. ${ }^{1} \mathrm{H}$ NMR chemical shift differences of PGME amides of $\mathbf{1}$ and $\mathbf{2}$.

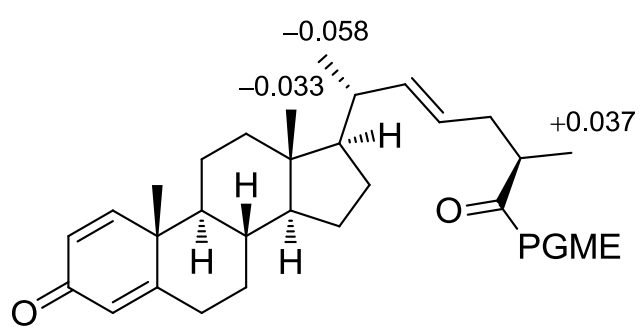

$\triangle \delta=\delta(S)-\delta(R)$ PGME amide

1a (S)-PGME

1 b (R)-PGME

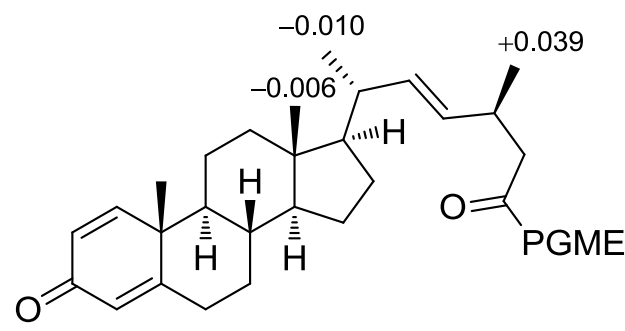

$\triangle \delta=\delta(R)-\delta(S)$ PGME amide

2a $(R)-P G M E$

2b (S)-PGME

The cytotoxicity of compounds 1-3 against HepG2, Hep3B, MDA-MB-231, MCF-7, and A-549 cancer cells was studied and shown in Table 3. Compound $\mathbf{3}$ showed potent cytotoxicity toward Hep3B, MDA-MB-231, MCF-7, and A-549 cancer cell lines, with $\mathrm{IC}_{50}$ values ranging from 2.05 to $2.83 \mu \mathrm{g} / \mathrm{mL}$. We also investigated the inhibition of these compounds toward LPS-induced pro-inflammatory protein (iNOS and COX-2) expression in RAW264.7 macrophage cells by Western blot analysis. At a concentration of $10 \mu \mathrm{M}$, compounds 2 and 3 reduced the levels of iNOS to $63.9 \pm 6.3 \%$ and $53.5 \pm 8.6 \%$, respectively; whereas, compound 2 enhanced the expression of COX-2 $(130.5 \pm 9.8 \%)$ in comparison with those of control cells stimulated with LPS only $(100 \%$ for both iNOS and COX-2). Also, compound 3 could inhibit the expression of iNOS protein but did not induce cytotoxicity in macrophage cells as determined through internal control $\beta$-actin expression, as shown in Figure 3. These results indicate that $\mathbf{3}$ possesses moderate anti-inflammatory activity and potent cytotoxicity, and might be useful for further medicinal study.

Table 3. Cytotoxicity data of compounds $\mathbf{1}-\mathbf{3}$.

\begin{tabular}{cccccc}
\hline \multirow{2}{*}{ Compound } & \multicolumn{5}{c}{ Cell lines IC $_{\mathbf{5 0}}(\boldsymbol{\mu g} / \mathbf{m L})$} \\
\cline { 2 - 6 } & Hep G2 & Hep 3B & MDA-MB-231 & MCF-7 & A549 \\
\hline $\mathbf{1}$ & 15.21 & - & 19.66 & - & - \\
$\mathbf{2}$ & 19.77 & - & - & - & - \\
$\mathbf{3}$ & 13.57 & 2.83 & 2.25 & 2.23 & 2.05 \\
doxorubicin & 0.31 & 0.40 & 1.32 & 0.68 & 1.33 \\
\hline
\end{tabular}

$(-)$ : Compound is considered inactive with $\mathrm{IC}_{50}>20 \mu \mathrm{g} / \mathrm{mL}$. 
Figure 3. Effect of compounds 1-3 at $10 \mu \mathrm{M}$ on the LPS-induced pro-inflammatory iNOS and on COX-2 protein expression of RAW264.7 macrophage cells by immunoblot analysis (A) Quantification of immunoblots of iNOS; (B) Quantification of immunoblots of COX-2. The values are means \pm SEM $(n=6)$. The relative intensity of the LPS alone stimulated group was taken as $100 \%$. * Significantly different from LPS alone stimulated group (* $P<0.05)$. ${ }^{\text {a }}$ Stimulated with LPS. ${ }^{\mathrm{b}}$ Stimulated with LPS in the presence of $\mathbf{1}-\mathbf{3}(10 \mu \mathrm{M})$; (C) Quantification of immunoblots of $\beta$-actin.

(A)

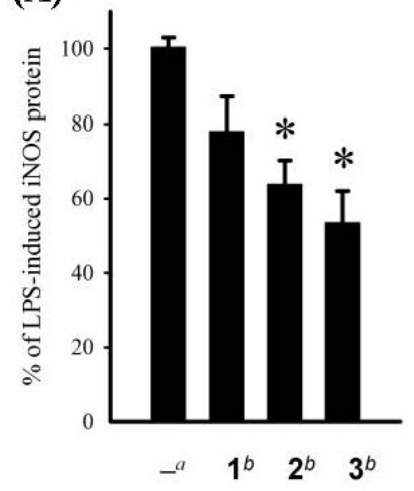

(B)

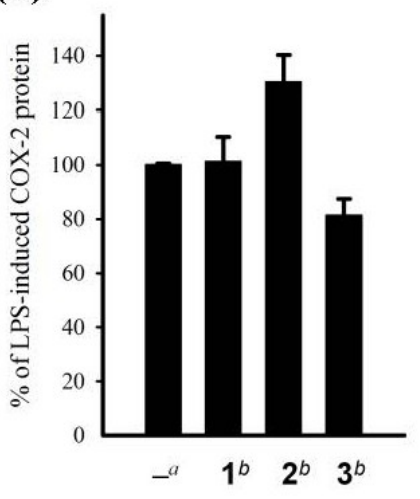

(C)

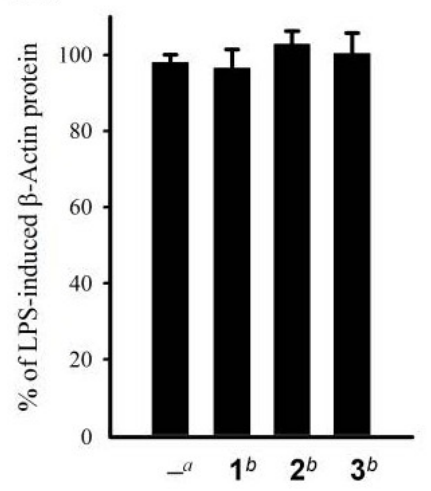

\section{Experimental Section}

\subsection{General Experimental Procedures}

Optical rotations were determined with a JASCO P1020 digital polarimeter. The IR spectra were obtained on a JASCO FT/IR-4100 spectrophotometer. The NMR spectra were recorded on a Bruker AVANCE 300 FT-NMR (or Varian MR-400 NMR) instrument at $300 \mathrm{MHz}$ (or $400 \mathrm{MHz}$ ) for ${ }^{1} \mathrm{H}$ (referenced to TMS for both $\mathrm{CDCl}_{3}$ and $\mathrm{C}_{5} \mathrm{D}_{5} \mathrm{~N}$ ) and $75 \mathrm{MHz}$ (or $100 / 125 \mathrm{MHz}$ ) for ${ }^{13} \mathrm{C}$ (referenced to $\delta_{\mathrm{C}} 77.0$ for $\mathrm{CDCl}_{3}$ and to internal TMS for $\mathrm{C}_{5} \mathrm{D}_{5} \mathrm{~N}$ ). ESIMS were recorded by ESI FT-MS on a Bruker APEX II mass spectrometer. Silica gel 60 (230-400 mesh, Merck, Darmstadt, Germany) and LiChroprep RP-18 (Merck, 40-63 $\mu \mathrm{m}$ ) were used for column chromatography. Precoated silica gel plates (Kieselgel 60 F254, 0.25 mm, Merck, Darmstadt, Germany) and precoated RP-18 F254S plates (Merck, Darmstadt, Germany) were used for TLC analyses. High-performance liquid chromatography was performed on a Hitachi L-7100 pump equipped with a Hitachi L-7400 UV detector at $210 \mathrm{~nm}$ and a semi-preparative reversed-phase column (Hibar Purospher RP-18e, $5 \mu \mathrm{m}, 250 \times 10 \mathrm{~mm}$, Merck, Darmstadt, Germany).

\subsection{Animal Material}

The soft coral $P$. acronocephala was collected by scuba divers, off the western coast of Pingtung county, in May 2009, at a depth of $10 \mathrm{~m}$, and was stored in a freezer until being extracted. This soft coral was identified by Prof. Chang-Fong Dai, Institute of Oceanography, National Taiwan University. A voucher specimen (specimen No. 200905PA) was deposited in the Department of Marine Biotechnology and Resources, National Sun Yat-sen University. 


\subsection{Extraction and Isolation}

The soft coral P. acronocephala (3.8 kg fresh wt) was collected and freeze-dried. The freeze-dried material was minced and extracted exhaustively with EtOH $(6 \times 2$ L $)$. The organic extract was concentrated to an aqueous suspension and was further partitioned between EtOAc and water. The EtOAc extract (30 g) was fractionated by open column chromatography on silica gel using $n$-hexane-EtOAc and EtOAc-MeOH mixtures of increasing polarity to yield 28 fractions. Fraction 21 (3.6 g), eluted with $n$-hexane-EtOAc (1:6), was further separated by silica gel column chromatography with gradient elution ( $n$-hexane-acetone, $5: 1$ to $2: 1$ ) to yield eight subfractions (21A to $21 \mathrm{H}$ ). Subfraction 21D was fractionated by RP-18 open column $\left(\mathrm{MeOH}-\mathrm{H}_{2} \mathrm{O}, 50 \%\right.$ to $\left.100 \%\right)$ to afford six subfractions (21D1 to 21D6). Compounds 1 (1.9 mg) and $2(2.6 \mathrm{mg})$ were obtained from subfraction $21 \mathrm{D} 5$ using RP-18 HPLC (MeOH- $\mathrm{H}_{2} \mathrm{O}$, gradient 75\% to 85\%). Compound 3 (5.1 mg) was obtained from fraction $25(0.85 \mathrm{~g})$ using repeatedly column chromatography over silica gel ( $n$-hexane-EtOAc, $1: 3$ to $0: 1)$ and RP-18 gel $\left(\mathrm{MeOH}-\mathrm{H}_{2} \mathrm{O}, 50 \%\right.$ to $\left.100 \%\right)$, and subsequently separated by RP-18 HPLC $\left(\mathrm{MeOH}-\mathrm{H}_{2} \mathrm{O}\right.$, gradient, $\left.65 \%-75 \%\right)$.

Paraminabic acid A (1): amorphous solid; $[\alpha]^{24}{ }_{\mathrm{D}}+13\left(c 0.09, \mathrm{CHCl}_{3}\right)$; IR (KBr) $v_{\max } 3400-2600$ (br), 2933, 2868, 2853, 1718, 1662, 1615, 1602, 1457, 1406, 1375, 1292, $1241 \mathrm{~cm}^{-1} ;{ }^{13} \mathrm{C}$ NMR and ${ }^{1} \mathrm{H}$ NMR data, see Tables 1 and 2; Selected ${ }^{1} \mathrm{H}$ NMR $\left(\mathrm{C}_{5} \mathrm{D}_{5} \mathrm{~N}, 400 \mathrm{MHz}\right)$ of $1: \delta 7.01(1 \mathrm{H}, \mathrm{d}, J=10.0 \mathrm{~Hz}, \mathrm{H}-1)$, $6.42(1 \mathrm{H}, \mathrm{dd}, J=10.0,2.0 \mathrm{~Hz}, \mathrm{H}-2), 6.26(1 \mathrm{H}, \mathrm{s}, \mathrm{H}-4), 5.50(1 \mathrm{H}, \mathrm{dt}, J=15.6,6.4 \mathrm{~Hz}, \mathrm{H}-23), 5.44(1 \mathrm{H}$, $\mathrm{dd}, J=15.6,8.8 \mathrm{~Hz}, \mathrm{H}-22), 2.78$ (1H, m, H-25), 2.65 (1H, m, H-24a), 2.40 (1H, m, H-24b), 1.38 (3H, d, $\left.J=6.8 \mathrm{~Hz}, \mathrm{H}_{3}-27\right), 1.10\left(3 \mathrm{H}, \mathrm{s}, \mathrm{H}_{3}-19\right), 1.03\left(3 \mathrm{H}, \mathrm{d}, J=6.4 \mathrm{~Hz}, \mathrm{H}_{3}-21\right), 0.67$ (3H, s, $\left.\mathrm{H}_{3}-18\right)$; ESIMS $m / z$ $433[\mathrm{M}+\mathrm{Na}]^{+}$; HRESIMS $m / z$ 433.2715 [M+ Na] $]^{+}$(calcd for $\mathrm{C}_{27} \mathrm{H}_{38} \mathrm{O}_{3} \mathrm{Na}, 433.2718$ ).

Paraminabic acid B (2): amorphous solid; $[\alpha]^{24}+60\left(c 0.16, \mathrm{CHCl}_{3}\right)$; IR (KBr) $v_{\max } 3400-2600$ (br), 2934, 2868, 1718, 1662, 1617, 1601, 1455, 1405, 1375, 1292, $1241 \mathrm{~cm}^{-1} ;{ }^{13} \mathrm{C}$ NMR and ${ }^{1} \mathrm{H}$ NMR data, see Tables 1 and 2; ${ }^{1} \mathrm{H}$ NMR $\left(\mathrm{C}_{5} \mathrm{D}_{5} \mathrm{~N}, 400 \mathrm{MHz}\right)$ of $2: \delta 7.01(1 \mathrm{H}, \mathrm{d}, J=10.0 \mathrm{~Hz}, \mathrm{H}-1), 6.42(1 \mathrm{H}, \mathrm{dd}$, $J=10.0,1.6 \mathrm{~Hz}, \mathrm{H}-2), 6.27(1 \mathrm{H}, \mathrm{s}, \mathrm{H}-4), 5.51(1 \mathrm{H}, \mathrm{dd}, J=15.2,7.2 \mathrm{~Hz}, \mathrm{H}-23), 5.41(1 \mathrm{H}, \mathrm{dd}, J=15.2$, $8.4 \mathrm{~Hz}, \mathrm{H}-22), 2.97$ (1H, m, H-24), 2.63 (1H, dd, $J=14.8,7.6 \mathrm{~Hz}, \mathrm{H}-25 \mathrm{a}), 2.55$ (1H, dd, J= 14.8, 7.2 Hz, H-25b), 2.31 (1H, td, $J=13.6,4.4$ Hz, H-6a), 2.18 (1H, dt, $J=13.6,2.4$ Hz, H-6b), 2.04 (1H, m, H-20), 1.90 (1H, dt, $J=12.4,3.2 \mathrm{~Hz}, \mathrm{H}-12 \mathrm{a}), 1.71$ (1H, m, H-16a), 1.70 (1H, m, H-7a), 1.54 (2H, m, H $\left.\mathrm{H}_{2}-11\right)$, $1.42(1 \mathrm{H}, \mathrm{m}, \mathrm{H}-8), 1.39$ (1H, m, H-15a), $1.25(1 \mathrm{H}, \mathrm{m}, \mathrm{H}-16 \mathrm{~b}), 1.21\left(3 \mathrm{H}, \mathrm{d}, J=6.8 \mathrm{~Hz}, \mathrm{H}_{3}-27\right), 1.09(3 \mathrm{H}$, s, $\left.\mathrm{H}_{3}-19\right), 1.08$ (1H, m, H-17), 1.06 (1H, m, H-12b), 1.04 (3H, d, J=6.0 Hz, H $\left.{ }_{3}-21\right), 0.99$ (1H, m, $\mathrm{H}-15 \mathrm{~b}), 0.88$ (1H, m, H-9), 0.83 (1H, m, H-14), 0.82 (1H, m, H-7b), 0.67 (3H, s, H -18$) ;{ }^{13} \mathrm{C}$ NMR $\left(\mathrm{C}_{5} \mathrm{D}_{5} \mathrm{~N}, 100 \mathrm{MHz}\right)$ of 2: $\delta 185.9(\mathrm{C}, \mathrm{C}-3), 175.2(\mathrm{C}, \mathrm{C}-26), 169.2(\mathrm{C}, \mathrm{C}-5), 156.0(\mathrm{CH}, \mathrm{C}-1), 135.5(\mathrm{CH}$, C-22), 132.7 (CH, C-23), 127.7 (CH, C-2), $124.0(\mathrm{CH}, \mathrm{C}-4), 55.9(\mathrm{CH}, \mathrm{C}-17), 55.7$ (CH, C-14), 52.6 (CH, C-9), 43.7 (C, C-10), $43.1\left(\mathrm{CH}_{2}, \mathrm{C}-25\right), 42.7$ (C, C-13), $40.3(\mathrm{CH}, \mathrm{C}-20), 39.6\left(\mathrm{CH}_{2}, \mathrm{C}-12\right), 35.4$ (CH, C-8), $34.1(\mathrm{CH}, \mathrm{C}-24), 33.8\left(\mathrm{CH}_{2}, \mathrm{C}-7\right), 32.9\left(\mathrm{CH}_{2}, \mathrm{C}-6\right), 28.7\left(\mathrm{CH}_{2}, \mathrm{C}-16\right), 24.4\left(\mathrm{CH}_{2}, \mathrm{C}-15\right), 22.9$ $\left(\mathrm{CH}_{2}, \mathrm{C}-11\right), 20.9\left(\mathrm{CH}_{3}, \mathrm{C}-21\right), 20.8\left(\mathrm{CH}_{3}, \mathrm{C}-27\right), 18.7\left(\mathrm{CH}_{3}, \mathrm{C}-19\right), 12.3\left(\mathrm{CH}_{3}, \mathrm{C}-18\right)$; ESIMS $m / z 433$ $[\mathrm{M}+\mathrm{Na}]^{+}$; HRESIMS $m / z$ 433.2715 [M+Na] ${ }^{+}$(calcd for $\mathrm{C}_{27} \mathrm{H}_{38} \mathrm{O}_{3} \mathrm{Na}, 433.2718$ ).

Paraminabic acid C (3): amorphous solid; $[\alpha]^{24}+43\left(c 0.09, \mathrm{CHCl}_{3}\right)$; IR (KBr) $v_{\max } 3400-2600$ (br), 3419, 2967, 2936, 2870, 1714, 1660, 1616, 1599, 1456, 1442, 1375, 1295, 1240, $1161 \mathrm{~cm}^{-1} ;{ }^{13} \mathrm{C}$ NMR and ${ }^{1} \mathrm{H}$ NMR data, see Tables 1 and $2 ;{ }^{1} \mathrm{H}$ NMR $\left(\mathrm{C}_{5} \mathrm{D}_{5} \mathrm{~N}, 400 \mathrm{MHz}\right)$ of 3: $\delta 7.01(1 \mathrm{H}, \mathrm{d}, J=10.0 \mathrm{~Hz}$, 
H-1), $6.41(1 \mathrm{H}, \mathrm{dd}, J=10.0,2.0 \mathrm{~Hz}, \mathrm{H}-2), 6.25(1 \mathrm{H}, \mathrm{s}, \mathrm{H}-4), 5.73(1 \mathrm{H}, \mathrm{dt}, J=15.2,7.4 \mathrm{~Hz}, \mathrm{H}-23), 5.49$ (1H, dd, $J=15.2,8.4 \mathrm{~Hz}, \mathrm{H}-22), 3.00$ (1H, br d, $J=12.4 \mathrm{~Hz}, \mathrm{H}-12 \mathrm{a}), 2.47$ (1H, m, H-20), 2.41 (2H, m, $\left.\mathrm{H}_{2}-24\right), 2.25$ (1H, m, H-15a), 2.22 (1H, m, H-6a), 2.17 (1H, m, H-6b), 2.02 (1H, m, H-8), 1.98 (1H, m, H-16a), 1.92 (2H, m, H-7a and H-11a), 1.90 (1H, m, H-16b), 1.84 (1H, m, H-11b), 1.64 (1H, m, H-15b), $1.60(1 \mathrm{H}, \mathrm{m}, \mathrm{H}-17), 1.40\left(3 \mathrm{H}, \mathrm{s}, \mathrm{H}_{3}-26\right), 1.39\left(3 \mathrm{H}, \mathrm{d}, J=6.4 \mathrm{~Hz}, \mathrm{H}_{3}-21\right), 1.39\left(3 \mathrm{H}, \mathrm{s}, \mathrm{H}_{3}-27\right), 1.38(1 \mathrm{H}$, m, H-14), 1.22 (1H, m, H-12b), 1.06 (1H, m, H-9), 1.00 (3H, s, $\left.\mathrm{H}_{3}-19\right), 0.80$ (1H, m, H-7b); ${ }^{13} \mathrm{C}$ NMR $\left(\mathrm{C}_{5} \mathrm{D}_{5} \mathrm{~N}, 100 \mathrm{MHz}\right)$ of 3: $\delta 185.9(\mathrm{C}, \mathrm{C}-3), 176.9(\mathrm{C}, \mathrm{C}-18), 169.1$ (C, C-5), $155.9(\mathrm{CH}, \mathrm{C}-1), 139.3(\mathrm{CH}$, C-22), 127.7 (CH, C-2), 125.3 (CH, C-23), 124.2 (CH, C-4), $69.9(\mathrm{CH}, \mathrm{C}-25), 57.1$ (C, C-13), $56.4(\mathrm{CH}$, C-14), 56.0 (CH, C-17), 52.6 (CH, C-9), $48.2\left(\mathrm{CH}_{2}, \mathrm{C}-24\right), 43.7$ (C, C-10), $42.0(\mathrm{CH}, \mathrm{C}-20), 37.5(\mathrm{CH}$, C-8), $37.2\left(\mathrm{CH}_{2}, \mathrm{C}-12\right), 33.8\left(\mathrm{CH}_{2}, \mathrm{C}-7\right), 32.8\left(\mathrm{CH}_{2}, \mathrm{C}-6\right), 30.5\left(\mathrm{CH}_{2}, \mathrm{C}-16\right), 29.9\left(\mathrm{CH}_{3}, \mathrm{C}-27\right), 29.7$ $\left(\mathrm{CH}_{3}, \mathrm{C}-26\right), 25.5\left(\mathrm{CH}_{2}, \mathrm{C}-15\right), 25.2\left(\mathrm{CH}_{2}, \mathrm{C}-11\right), 21.0\left(\mathrm{CH}_{3}, \mathrm{C}-21\right), 18.7\left(\mathrm{CH}_{3}, \mathrm{C}-19\right)$; ESIMS $m / z 449$ $[\mathrm{M}+\mathrm{Na}]^{+}$; HRESIMS $m / z$ 449.2666 [M+Na] $]^{+}$(calcd for $\mathrm{C}_{27} \mathrm{H}_{38} \mathrm{O}_{4} \mathrm{Na}, 449.2668$ ).

\subsection{Preparation of (S) and (R)-PGME amides of $\mathbf{1}$ and $\mathbf{2}$}

To a stirred solution of compound $1(0.5 \mathrm{mg})$ and $(S)$-PGME $(2 \mathrm{mg})$ in a $1 \mathrm{~mL}$ mixture of $\mathrm{CHCl}_{3}$-DMF (10:1) were successively added DMAP (2 mg) and 4-DMAP·HCl (2 mg) [5]. After the mixture was stirred at $0{ }^{\circ} \mathrm{C}$ for $5 \mathrm{~min}, \mathrm{EDC} \cdot \mathrm{HCl}(2 \mathrm{mg})$ was added. The reaction mixture was then moved to a refrigerator at $4{ }^{\circ} \mathrm{C}$ for overnight. The mixture was then stirred at room temperature for $3 \mathrm{~h}$. Subsequently, ethyl acetate was added, and the resulting solution was successively washed with $5 \%$ $\mathrm{HCl}$, saturated $\mathrm{NaHCO}_{3}$ (aq), and brine. The organic layer was dried over anhydrous $\mathrm{Na}_{2} \mathrm{SO}_{4}$ and concentrated to give a residue, which was chromatographed on silica gel using $n$-hexane-EtOAc (5:1) as eluent to afford the $(S)$-PGME amide (1a) $(0.3 \mathrm{mg})$. The same procedure was used to prepare the $(R)$-PGME amide (1b) (0.3 mg from $0.5 \mathrm{mg}$ of 1) with $(R)$-PGME. Selective ${ }^{1} \mathrm{H} \mathrm{NMR}\left(\mathrm{CDCl}_{3}, 300 \mathrm{MHz}\right)$ of 1a: $\delta 7.343(5 \mathrm{H}$, br s, Ph), $7.049(1 \mathrm{H}, \mathrm{d}, J=10.2 \mathrm{~Hz}, \mathrm{H}-1), 6.384(1 \mathrm{H}, \mathrm{d}, J=7.0 \mathrm{~Hz}, \mathrm{NH}), 6.228(1 \mathrm{H}$, d, $J=10.2 \mathrm{~Hz}, \mathrm{H}-2), 6.068$ (1H, s, H-4), $5.586(1 \mathrm{H}, \mathrm{d}, J=7.0 \mathrm{~Hz}, \mathrm{CH}-\mathrm{N}), 5.207$ (2H, overlapped, H-22 and $\mathrm{H}-23), 3.730(3 \mathrm{H}, \mathrm{s}, \mathrm{OMe}), 1.225\left(3 \mathrm{H}, \mathrm{s}, \mathrm{H}_{3}-19\right), 1.143\left(3 \mathrm{H}, \mathrm{d}, J=6.3 \mathrm{~Hz}, \mathrm{H}_{3}-27\right), 0.911(3 \mathrm{H}, \mathrm{d}$, $\left.J=6.4 \mathrm{~Hz}, \mathrm{H}_{3}-21\right), 0.711\left(3 \mathrm{H}, \mathrm{s}, \mathrm{H}_{3}-18\right)$; selective ${ }^{1} \mathrm{H} \mathrm{NMR}\left(\mathrm{CDCl}_{3}, 300 \mathrm{MHz}\right)$ of $\mathbf{1 b}: \delta 7.345(5 \mathrm{H}$, br s, $\mathrm{Ph}), 7.052(1 \mathrm{H}, \mathrm{d}, J=9.7 \mathrm{~Hz}, \mathrm{H}-1), 6.405(1 \mathrm{H}, \mathrm{d}, J=7.4 \mathrm{~Hz}, \mathrm{NH}), 6.226(1 \mathrm{H}, \mathrm{d}, J=9.7 \mathrm{~Hz}, \mathrm{H}-2), 6.066$ $(1 \mathrm{H}, \mathrm{s}, \mathrm{H}-4), 5.568(1 \mathrm{H}, \mathrm{d}, J=7.4 \mathrm{~Hz}, \mathrm{CH}-\mathrm{N}), 5.286(2 \mathrm{H}$, overlapped, $\mathrm{H}-22$ and $\mathrm{H}-23), 3.726(3 \mathrm{H}, \mathrm{s}$, $\mathrm{OMe}$ ), 1.229 (3H, s, $\left.\mathrm{H}_{3}-19\right), 1.106\left(3 \mathrm{H}, \mathrm{d}, J=6.0 \mathrm{~Hz}, \mathrm{H}_{3}-27\right), 0.969$ (3H, d, $\left.J=6.5 \mathrm{~Hz}, \mathrm{H}_{3}-21\right), 0.744$ $\left(3 \mathrm{H}, \mathrm{s}, \mathrm{H}_{3}-18\right)$. The same procedure was applied on $2(0.5 \mathrm{mg})$ to prepare the $(R)$-PGME amide 2a $(0.4 \mathrm{mg})$ and the $(S)$-PGME amide $2 \mathrm{a}(0.4 \mathrm{mg}$ from $0.5 \mathrm{mg}$ of 2$)$. Selective ${ }^{1} \mathrm{H} \mathrm{NMR}\left(\mathrm{CDCl}_{3}, 300 \mathrm{MHz}\right)$ of $2 \mathbf{b}: \delta 7.357(5 \mathrm{H}$, br s, Ph), $7.050(1 \mathrm{H}, \mathrm{d}, J=10.1 \mathrm{~Hz}, \mathrm{H}-1), 6.362(1 \mathrm{H}, \mathrm{d}, J=7.4 \mathrm{~Hz}, \mathrm{NH}), 6.224(1 \mathrm{H}$, d, $J=10.1 \mathrm{~Hz}, \mathrm{H}-2), 6.067$ (1H, s, H-4), 5.597 (1H, d, $J=7.4 \mathrm{~Hz}, \mathrm{CH}-\mathrm{N}), 5.241$ (2H, overlapped, H-22 and $\mathrm{H}-23), 3.727(3 \mathrm{H}, \mathrm{s}, \mathrm{OMe}), 1.225\left(3 \mathrm{H}, \mathrm{s}, \mathrm{H}_{3}-19\right), 1.016\left(3 \mathrm{H}, \mathrm{d}, J=6.6 \mathrm{~Hz}, \mathrm{H}_{3}-27\right), 0.947(3 \mathrm{H}, \mathrm{d}$, $\left.J=6.5 \mathrm{~Hz}, \mathrm{H}_{3}-21\right), 0.716\left(3 \mathrm{H}, \mathrm{s}, \mathrm{H}_{3}-18\right)$; selective ${ }^{1} \mathrm{H} \mathrm{NMR}\left(\mathrm{CDCl}_{3}, 300 \mathrm{MHz}\right)$ of $2 \mathrm{a}: \delta 7.341(5 \mathrm{H}$, br s, $\mathrm{Ph}), 7.051(1 \mathrm{H}, \mathrm{d}, J=10.2 \mathrm{~Hz}, \mathrm{H}-1), 6.455(1 \mathrm{H}, \mathrm{d}, J=6.9 \mathrm{~Hz}, \mathrm{NH}), 6.223(1 \mathrm{H}, \mathrm{d}, J=10.2 \mathrm{~Hz}, \mathrm{H}-2)$, $6.066(1 \mathrm{H}, \mathrm{s}, \mathrm{H}-4), 5.579(1 \mathrm{H}, \mathrm{d}, J=6.9 \mathrm{~Hz}, \mathrm{CH}-\mathrm{N}), 5.238(2 \mathrm{H}$, overlapped, $\mathrm{H}-22$ and $\mathrm{H}-23), 3.722(3 \mathrm{H}$, s, OMe), $1.226\left(3 \mathrm{H}, \mathrm{s}, \mathrm{H}_{3}-19\right), 0.977\left(3 \mathrm{H}, \mathrm{d}, J=6.2 \mathrm{~Hz}, \mathrm{H}_{3}-27\right), 0.957$ (3H, d, $\left.J=6.0 \mathrm{~Hz}, \mathrm{H}_{3}-21\right), 0.722$ $\left(3 \mathrm{H}, \mathrm{s}, \mathrm{H}_{3}-18\right)$. It has to be noted that the chemical shifts of $\mathrm{H}-22$ and $\mathrm{H}-23$ in both PGME amides of 1 and 2 were overlapped seriously, that might interfere the correct assignment of the corresponding 
protons. Fortunately, we afford the $\Delta \delta$ values of the $\mathrm{H}_{3}-21$ and $\mathrm{H}_{3}-18$ of $(S)$ and $(R)$-PGME amides of both $\mathbf{1}$ and $\mathbf{2}$ which could be used for configuration assignment of C-25 in $\mathbf{1}$ and C-24 in $\mathbf{2}$, respectively.

\subsection{Cytotoxicity Testing}

Cell lines were purchased from the American Type Culture Collection (ATCC). Compounds were assayed for cytotoxicity against human liver carcinoma (HepG2 and HepG3), human breast carcinoma (MCF-7 and MDA-MB-231), and human lung carcinoma (A-549) cells using the 3-(4,5-dimethylthiazol-2-yl)-2,5-diphenyltetrazolium bromide (MTT) method [8]. Freshly trypsinized cell suspensions were seeded in 96-well microtiter plates at densities of 5000-10,000 cells per well with tested compounds added from DMSO-diluted stock. After 3 days in culture, attached cells were incubated with MTT $(0.5 \mathrm{mg} / \mathrm{mL}, 1 \mathrm{~h})$ and subsequently dissolved in DMSO. The absorbency at $550 \mathrm{~nm}$ was then measured using a microplate reader. $\mathrm{The} \mathrm{IC}_{50}$ is the concentration of agent that reduced cell growth by $50 \%$ under the experimental conditions.

\subsection{In Vitro Anti-Inflammatory Assay}

Macrophage (RAW264.7) cell was purchased from ATCC. In vitro anti-inflammatory activities of tested compounds were measured by examining the inhibition of lipopolysaccharide (LPS) induced upregulation of iNOS and COX-2 proteins in macrophage cells using Western blotting analysis [9,10].

\section{Conclusions}

Our previous investigation on $P$. acronocephala has successfully discovered marine withanolides with potent anti-inflammatory activity. In this study, we reported three steroidal carboxylic acids, of which 3 exhibited potent cytotoxicity toward Hep3B, MDA-MB-231, MCF-7, and A-549 cancer cell lines. Compound 2, the second member of 27-norergostan-26-oic acid obtained from nature [11,12], was isolated from the soft coral for the first time. Our present investigation demonstrated that the soft coral, $P$. acronocephala, is a useful source for the discovery of bioactive substances.

\section{Acknowledgments}

This work was supported by grants from the National Science Council of Taiwan (NSC100-2320-B-110-001-MY2) and Ministry of Education (00C030205) awarded to J.-H. Sheu.

\section{References}

1. Chao, C.-H.; Chou, K.-J.; Wen, Z.-H.; Wang, G.-H.; Wu, Y.-C.; Dai, C.-F.; Sheu, J.-H. Paraminabeolides A-F, cytotoxic and anti-inflammatory marine withanolides from the soft coral Paraminabea acronocephala. J. Nat. Prod. 2011, 74, 1132-1141.

2. Ksebati, M.B.; Schmitz, F.J. Minabeolides: A group of withanolides from a soft coral, Minabea sp. J. Org. Chem. 1988, 53, 3926-3929. 
3. Su, B.-N.; Park, E.K.; Nikolic, D.; Santarsiero, B.D.; Mesecar, A.D.; Vigo, J.S.; Graham, J.G.; Cabieses, F.; van Breemen, R.B.; Fong, H.H.S.; et al. Activity-guided isolation of novel norwithanolides from Deprea subtriflora with potential cancer chemopreventive activity. J. Org. Chem. 2003, 68, 2350-2361.

4. Wang, W.; Lee, J.S.; Nakazawa, T.; Ukai, K.; Mangindaan, R.E.P.; Wewengkang, D.S.; Rotinsulu, H.; Kobayashi, H.; Tsukamoto, S.; Namikoshi, M. (25S)-Cholesten-26-oic acid derivatives from an Indonesian soft coral Minabea sp. Steroids 2009, 74, 758-760.

5. Chao, C.-H.; Wen, Z.-H.; Chen, I.-M.; Su, J.-H.; Huang, H.-C.; Chiang, M.Y.; Sheu, J.-H. Anti-inflammatory steroids from the octocoral Dendronephthya griffini. Tetrahedron 2008, 64, 3554-3560.

6. Nagai, Y.; Kusumi, T. New chiral anisotropic reagents for determining the absolute configuration of carboxylic acids. Tetrahedron Lett. 1995, 36, 1853-1856.

7. Yabuuchi, T.; Kusumi, T. Phenylglycine methyl ester, a useful tool for absolute configuration determination of various chiral carboxylic acids. J. Org. Chem. 2000, 65, 397-404.

8. Alley, M.C.; Scudiero, D.A.; Monks, A.; Hursey, M.L.; Czerwinski, M.J.; Fine, D.L; Abbott, B.J.; Mayo, J.G.; Shoemaker, R.H.; Boyd, M.R. Feasibility of drug screening with panels of human tumor cell lines using a microculture tetrazolium assay. Cancer Res. 1988, 48, 589-601.

9. Jean, Y.-H.; Chen, W.-F.; Sung, C.-S.; Duh, C.-Y.; Huang, S.-Y.; Lin, C.-S.; Tai, M.-H.; Tzeng, S.-F.; Wen, Z.-H. Capnellene, a natural marine compound derived from soft coral, attenuates chronic constriction injury-induced neuropathic pain in rats. Br. J. Pharmacol. 2009, 158, 713-725.

10. Wen, Z.-H.; Chao, C.-H.; Sheu, J.-H. A neuroprotective sulfone of marine origin and the in vivo anti-inflammatory activity of an analogue. Eur. J. Med. Chem. 2010, 45, 5998-6004.

11. Finamore, E.; Minale, L.; Riccio, R.; Rinaldo, G.; Zollo, F. Novel marine polyhydroxylated steroids from the starfish Myxoderma platyacanthum. J. Org. Chem. 1991, 56, 1146-1153.

12. Sarma, N.S.; Krishna, M.S.; Pasha, S.G.; Prakasa Rao, T.S.; Venkateswarlu, Y.; Parameswaran, P.S. Marine metabolites: The sterols of soft coral. Chem. Rev. 2009, 109, 2803-2828.

Samples Availability: Not available.

(C) 2013 by the authors; licensee MDPI, Basel, Switzerland. This article is an open access article distributed under the terms and conditions of the Creative Commons Attribution license (http://creativecommons.org/licenses/by/3.0/). 